Research Paper

\title{
Association between HLA-B*46 Allele and Graves Disease in Asian Populations: A Meta-Analysis
}

\author{
Yiping $\mathrm{Li}^{1,2}$, Yufeng $\mathrm{Yao}^{3}$, Man Yang², Li Shi ${ }^{3}$, Xianli $\mathrm{Li}^{2}$, Ying Yang ${ }^{2}$, Ying Zhang² and Chunjie Xiao ${ }^{\bowtie}$ \\ 1. School of Medicine, Yunnan University, Kunming 650091, Yunnan, China; \\ 2. Department of Endocrinology and Metabolism, The second people's hospital of Yunnan province, Kunming 650021, Yunnan, China; \\ 3. Institute of Medical Biology, Chinese Academy of Medical Sciences \& Peking Union Medical College, Kunming 650118, Yunnan, China.
}

$\triangle$ Corresponding author: Prof. Chunjie Xiao, School of Medicine, Yunnan University, Kunming 650091, Yunnan, China. Email: chjxiao@ynu.edu.cn.

( ) Ivyspring International Publisher. This is an open-access article distributed under the terms of the Creative Commons License (http://creativecommons.org/ licenses/by-nc-nd/3.0/). Reproduction is permitted for personal, noncommercial use, provided that the article is in whole, unmodified, and properly cited.

Received: 2012.09.03; Accepted: 2012.12.28; Published: 2013.01.03

\begin{abstract}
Graves' disease (GD) is a leading cause of hyperthyroidism, which affects $1.0-1.6 \%$ of the general population. Previous studies reported a higher GD prevalence in Asian populations compared to Caucasian populations. The etiology of GD involves complex interactions between predisposing genes and environmental triggers. Genetic studies have shown that the human leukocyte antigen (HLA) is an important candidate genetic region associated with GD in Asian populations. However, the results were inconsistent and inconclusive. Here, we performed a meta-analysis to evaluate the role of the HLA-B*46 allele in GD in Asian populations. A total of 14 case-controlled studies on the association of the HLA-B*46 allele in 1743 GD patients and 5689 controls were included. Our results showed a trend toward an increased risk of GD in HLA-B*46-positive subjects compared to those HLA-B*46-negative $(\mathrm{OR}=2.48 ; 95 \% \mathrm{Cl}=1.96-3.13, \mathrm{P}<0.0 \mathrm{I})$. However, there were some limitations to the current meta-analysis, such as heterogeneity $\left(P_{\text {heterogeneity }}<0.0 \mathrm{I}\right.$ and $\left.\mathrm{I}^{2}=68.0 \%\right)$ or the different typing methods (serological and genotyping methods). The meta-analysis indicated that the HLA-B*46 allele is a risk factor for GD in Asian populations. Future studies on the role of the HLA-B*46 allele in GD should consider complications such as periodic paralysis, ophthalmopathy and recurrence.
\end{abstract}

Key words: HLA-B*46; Graves' disease; Meta-analysis; Asian populations.

\section{Introduction}

Graves' disease (GD) is a leading cause of hyperthyroidism, which is characterized by diffuse goiter and thyroid-specific auto-antibodies, with/ without ophthalmopathy and/or dermopathy [1]. The disease affects $1.0-1.6 \%$ of the general population with a strong preponderance of females [2,3]. Besides the sex difference in GD prevalence, evidence has indicated a prevalence of GD in Asian populations compared to Caucasian populations [4].

The etiology of GD is incompletely understood, but seems to involve complex interactions between predisposing genes and environmental triggers $[5,6]$.
Genetic studies have shown that the human leukocyte antigen (HLA) region on chromosome 6p21.31 is an important candidate genetic region associated with GD, as the HLA region is highly polymorphic and contains many immune response genes. This region encodes genes that are grouped into three classes: class I genes (HLA-A, HLA-B and HLA-C) class II genes (HLA-DR, HLA-DP and HLA-DQ) and class III genes. Many studies regarding HLA allelic associations with GD have been performed, and different associations of GD and HLA alleles have been reported among diverse ethnic populations. For exam- 
ple, HLA-B*08 (HLA class I), HLA-DR3 and HLA-DQA1*05:01 (HLA class II) are associated with a high risk of GD, and HLA-DRB1*07:01 is a protective allele against GD in Caucasian populations [7-11]. However, the Caucasian GD-associated HLA alleles are absent or occur in low frequencies in Asian populations. In Asian populations, HLA-B ${ }^{*} 46$, HLA-DRB1*08:03, HLA-DRB1*09:01 and HLA-DRB1*14:03 alleles were reported as risk factors of GD [12-25]. However, the results are somewhat contradictory [12-27], which may be due, in part, to the small sample sizes of the studies.

Meta-analysis is an effective statistical tool to combine data from previous studies to provide a more accurate estimate of cohort size effects and reduce the probability of false-negative results [28]. Therefore, meta-analysis of the association between HLA- ${ }^{*} 46$ and GD in Asian populations is necessary to clarify and evaluate the role of HLA-B*46 in GD.

In the current study, we collected 14 previous studies for a carefully designed meta-analysis to investigate the association of HLA-B ${ }^{*} 46$ with GD in Asian populations and provide a more thorough evaluation of the significance of this association.

\section{Methods}

\section{Statistics for meta-analysis}

\section{Search strategy}

All studies included in the present analysis explored the association between HLA-B*46 allele and
GD in Asian populations. Sources included MEDLINE, EMBASE, Science Citation Index Expanded (ISI Web of Science) and references of retrieved articles (up to March 2012). The search terms were as follows: 'HLA' OR 'human leukocyte antigen' AND 'Graves' disease'.

\section{Selection}

To be included in the meta-analysis, studies had to meet all the following criteria: (a) an unrelated case-control design, (b) related to Asian population (c) available serological typing, genotypic number or frequency, and (d) English language. If there were multiple publications from the same group, only the most recent study was included in the analysis. Duplicate reports were deleted.

\section{Data extraction}

Two authors who conducted the literature search (Yiping Li and Yufeng Yao) also independently extracted data from the studies. Any disagreement was adjudicated by consensus and consulting another two authors (Ying Zhang and Chunjie Xiao). The authors' names, journals and years of publication, regions of origin, diagnoses criteria of GD, cases and control sizes, and HLA-B ${ }^{*} 46$ antigen or gene frequencies were collected. Characteristics of each study were summarized in Table 1 . The antigen frequency $(F)$ was transformed into a gene frequency $(f)$ using the equation: $f=1-\sqrt{1-F}$ when only the antigen frequency was provided [29].

Table I. Characteristics of case-control studies included in current meta-analysis of the association between HLA-B*46 and GD.

\begin{tabular}{|c|c|c|c|c|c|c|c|c|c|c|}
\hline Study ID & Author & Year & Population & Number of GD, Age & Number of control, Age & GD & & Control & & Reference \\
\hline & & & & & & $\mathrm{B}^{*} 46(+)$ & $\mathrm{B}^{*} 46(-)$ & $B^{*} 46(+)$ & $B^{*} 46(-)$ & \\
\hline 001 & Chan SH & 1978 & Chinese & $86, \mathrm{NA}$ & 238, NA & 53 & 119 & 57 & 419 & [27] \\
\hline 002 & Hawkins BR & 1985 & Chinese & 132, NA & 110 , undergraduate & 86 & 178 & 31 & 189 & {$[12]$} \\
\hline 003 & Naito S & 1987 & Japanese & $61,35.9 \pm 11.8$ & 1998, NA & 15 & 107 & 172 & 3824 & {$[13]$} \\
\hline 004 & Tamai $\mathrm{H}$ & 1987 & Japanese & $35,19 \sim 54$ & 263, NA & 4 & 66 & 19 & 507 & [26] \\
\hline 005 & Yeo PP & 1989 & Chinese & 159, NA & 330, NA & 83 & 235 & 84 & 576 & {$[14]$} \\
\hline 006 & Inoue D & 1991 & Japanese & $88,34.8$ & 186, NA & 20 & 156 & 23 & 349 & {$[16]$} \\
\hline 007 & Dong RP & 1992 & Japanese & $76,32.4$ & 317, NA & 17 & 135 & 35 & 599 & [17] \\
\hline 008 & Chan SH & 1993 & Chinese & $33, \mathrm{NA}$ & 79, NA & 16 & 50 & 20 & 138 & {$[18]$} \\
\hline 009 & Onuma $\mathrm{H}$ & 1994 & Japanese & $106,28.3^{*}$ & 100, NA & 34 & 178 & 8 & 192 & [19] \\
\hline 010 & Cavan DA & 1994 & Chinese & $97, \mathrm{NA}$ & 105, NA & 58 & 136 & 36 & 174 & {$[20]$} \\
\hline 011 & Ohtsuka K & 1998 & Japanese & $94,13 \sim 69$ & 767, NA & 14 & 174 & 38 & 1496 & [21] \\
\hline 012 & Huang SM & 2003 & Chinese & 236, NA & 533, NA & 86 & 386 & 119 & 947 & {$[22]$} \\
\hline 013 & Cho WK & 2011 & Korean & $41, \mathrm{NA}$ & 159 , adults & 24 & 58 & 18 & 300 & {$[24]$} \\
\hline 014 & Chen PL & 2011 & Chinese & $499,41.9 \pm 12.4$ & $504,55.7 \pm 18.5$ & 173 & 825 & 137 & 871 & [23] \\
\hline
\end{tabular}

GD, Graves' disease; NA, not available; *the age was the onset of GD. 


\section{Meta Statistical analysis}

We compared GD cases with controls using the allelic model for HLA-B*46 (present/absent). The odds ratio (OR) and $95 \%$ confidence interval (CI) were calculated to determine the differences in each HLA-B ${ }^{*} 46$ between cases and controls. The $\chi^{2}$-based $Q$ statistic test was used for assessment of between-study heterogeneity [30], which was considered significant at a P-value $<0.10$. The inconsistency index $\left(\mathrm{I}^{2}\right)$ was calculated to evaluate the amount of variation caused by heterogeneity rather than by chance, where higher index values indicated the existence of heterogeneity [31]. Data were combined using fixed-effects (the Mantel-Haenszel method) or random-effects (the DerSimonian and Laird method) models. The fixed-effects model (if $\mathrm{P}>0.10$ ) or the random-effects model (if $\mathrm{P}<0.10$ ) was used to pool the results. From the individual ORs, a pooled OR was estimated, of which the statistical significance was determined using the Z-test. A P-value $<0.05$ was considered statistically significant. Sensitivity analysis was performed to assess the stability of these results. Potential publication bias and small study effect and/or publication bias was estimated by the Begg's funnel plot and Peters' test (A modification of Macaskill's test) $[32,33]$. A P-value $<0.05$ was considered to represent a statistically significant publication bias. All P-values were two-sided. All meta-analyses were performed using Stata statistical software (Stata/SE 12; Stata Corp., College Station, TX, USA).

\section{Results}

\section{Characteristics of including studies}

The initial search strategy to identify association studies for GD and HLA yielded a total of 182 potentially relevant references among all the databases, 84 of which were overlapping. As two studies included the same subjects, the one including more samples was chosen for the meta-analysis [15, 16]. A study on Chinese population from Singapore by the same research group [25, 27, 34] was replaced by another one including the largest number of subjects [27] for this meta-analysis. In total, 14 case-control studies of HLA-B $^{*} 46$ allelic polymorphisms with 1743 GD patients and 5689 controls were included [12-14, 16-24, $26,27]$. All subjects of the included studies were composed of Asian populations. Among the 14 studies, seven were from Chinese populations, six from Japanese populations and one from a Korean population. Of all included studies, 10 clearly noted the diagnosis of GD, which was based on clinical symptoms and biochemical hyperthyroidism and the remaining four did not note the diagnostic GD standard, but included cases considered as GD and in two studies, patients received treatment for GD (Table 1).

\section{Association of the HLA-B*46 allele with GD}

The ORs and $95 \%$ CIs of the association of GD with HLA-B*46 in each of studies are shown in Fig. 1. The heterogeneity test showed that there was significant heterogeneity among the results of individual studies ( $P_{\text {heterogeneity }}<0.01$ and $\mathrm{I}^{2}=68.0 \%$ for HLA-B*46). Therefore, the random effect model (D-L heterogeneity) was used for calculating the pooled ORs and 95\% CIs. Overall, there was a trend toward an increased risk of GD among HLA-B ${ }^{*} 46$ positive cases compared with those HLA-B ${ }^{*} 46$ negative $(\mathrm{OR}=$ 2.48; 95\% CI 1.96-3.13, P < 0.01). The meta-analysis standard forest plots are shown in Fig. 1.

\section{Publication bias}

Publication bias via Begg's funnel plot (Fig. 2) and small study effect and/or publication bias via Peters' test (A modification of Macaskill's test) were performed. The Begg's and Peters' test did not suggest publication bias and small study effect in current meta-analysis. The P-values of the Begg's and Peters' tests were 0.443 and 0.227 , respectively.

\section{Sensitivity analysis}

Sensitivity analyses were conducted to assess whether each individual study influenced the final results. Sensitivity analyses were performed by omitting one study at a time and calculating the pooled ORs for the remaining studies (Fig. 3). These analyses showed that none of the individual studies influenced the pooled ORs, which ranged from 2.30 (95\% CI = $1.86-2.83)$ to 2.58 (95\% CI $=1.99-3.34)$. The sensitivity analyses indicated that the results of the meta-analysis were reliable and stable.

\section{Discussion}

Several studies reported that the variation of HLA class I could trigger autoimmunity through presenting microbial antigens that are sufficiently similar to self-antigens to nucleated cells and activated autoreactive $\mathrm{T}$ cells that can cross-react with self-antigens [26-28]. For example, Yersinia enterocolitica, which is a key environmental trigger for GD, has been postulated to play a role in GD induction via molecular mimicry [26-28]. In addition, other studies have reported that the upregulation of HLA class I molecules on thyroid cells, including HLA-B and HLA-C in response to immune cell infiltration of the thyroid gland, may be one of the earliest features of autoimmune attack in GD [35]. The association stud- 
ies indicated that the HLA-B*46 was associated with GD in Asian populations. To evaluate the role of HLA-B ${ }^{*} 46$ in GD, we performed a meta-analysis on the association of HLA-B*46 with GD in Asian populations and our results indicated a positive association (OR $=2.48$; 95\% CI 1.96-3.13).

Our results were different from studies of Caucasian populations, which reported that HLA-B ${ }^{*} 08$ was associated with a high risk of GD (OR: 1.5-3.5) [36]. The reason for these different association results among different populations could be due to eth-

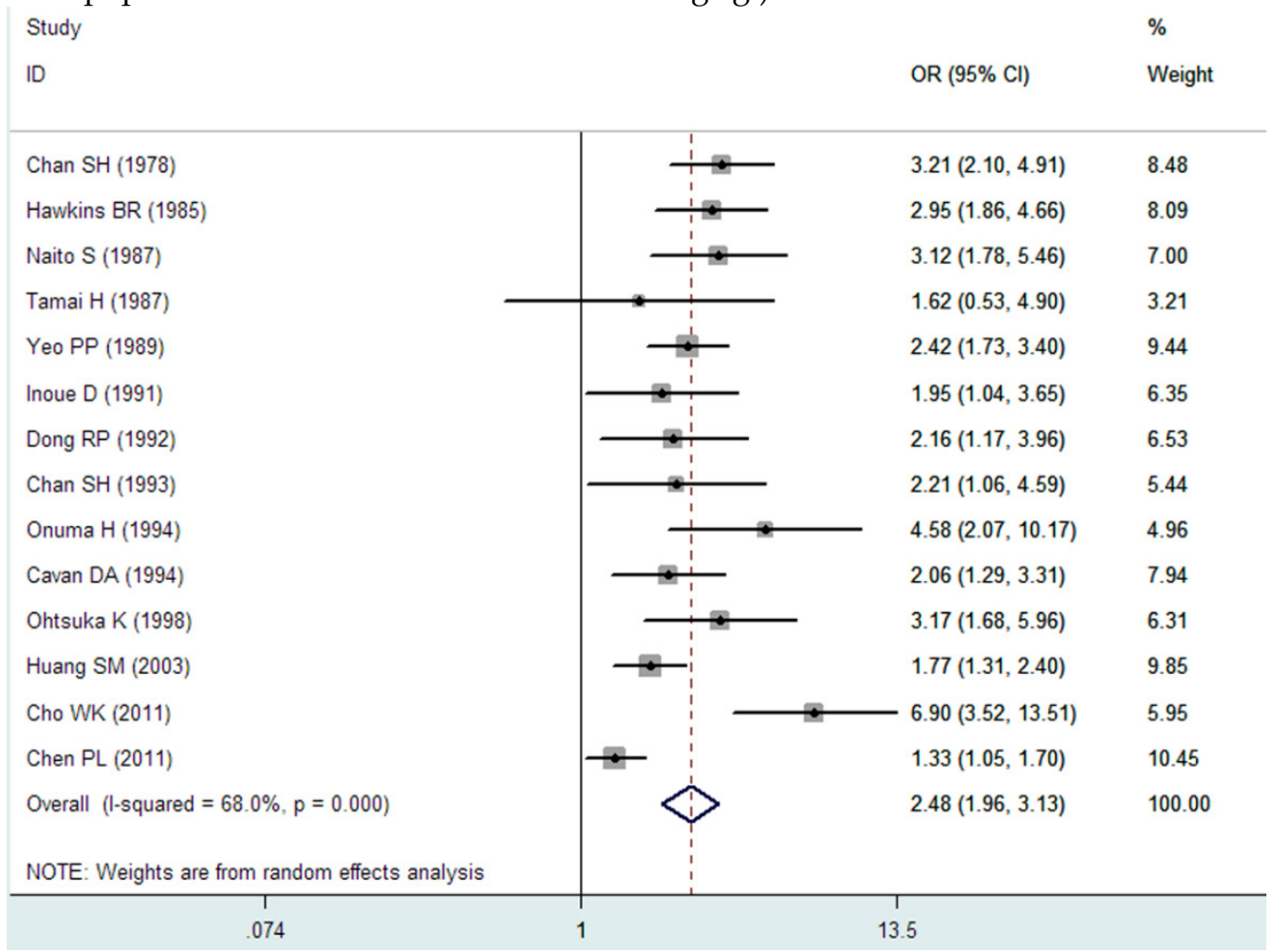

Figure I. Individual and pooled ORs and $95 \% \mathrm{Cl}$ for HLA-B*46 (positive vs. negative) for all studies.



Figure 2. Begg's funnel plot analysis for the comparison of the HLA-B*46. P value of Begg's test was 0.443 (continuity corrected). no-genetic differences. It is evident that the distribution of HLA-B ${ }^{*} 46$ and HLA-B ${ }^{*} 08$ are different between European and Asian populations. In European populations, the allelic frequency of HLA-B*08 is about $12 \%$, while the allelic frequency is $0.3 \sim 0.5 \%$ in most Asian populations (http://www.ncbi.nlm.nih. gov/projects/gv/mhc/ihwg.cgi). By contrast, the allelic frequency of HLA-B ${ }^{*} 46$ is $3.9 \sim 8.6 \%$ in Asian populations and almost zero in Europe populations (http://www.ncbi.nlm.nih.gov/projects/gv/mhc/ih wg.cgi). 


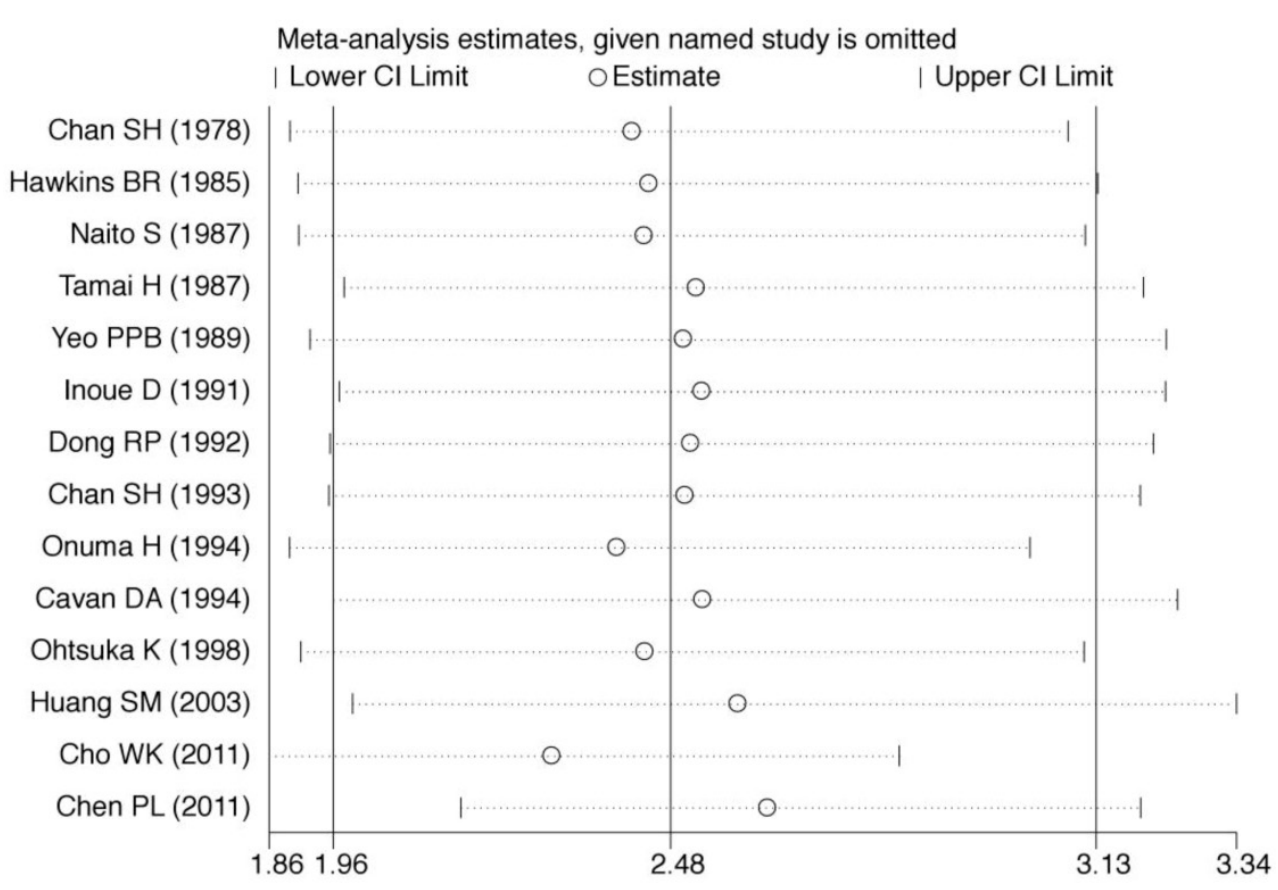

Figure 3. Sensitivity analysis was performed by omitting one study at a time and calculating the pooled ORs for the remaining studies.

Linkage disequilibrium (LD) refers to the non-random association between alleles at adjacent genetic loci. LD patterns across the human genome varies markedly between regions and populations [37-39], and is currently a subject of intense interest both as a mapping tool for the identification of disease susceptibility genes and for what they may reveal about a population's demographic history and the selective forces acting thereon. The global LD characteristics between HLA class I and II genes have been investigated for over 30 years [40-43], and the term 'ancestral haplotype' has been coined to describe conserved, population-specific haplotypes [44]. For example, in European populations, the HLA-B*08 and HLA-DRB*03 alleles had a stronger linkage disequilibrium and formed the ancestral haplotype 8.1 [45]. Interestingly, many studies reported that HLA-DRB*03:01 was strongly associated with GD from genetic studies [46]. Therefore, it is possible that HLA-B $^{*} 46$ was in a LD with a polymorphism locus of a HLA class II gene, which might influence professional antigen presenting cells through aberrant expression of HLA class II molecules on thyrocytes. However, in Asian populations, the HLA-B ${ }^{*} 46$ are partly in LD of the DRB1*08:03, HLA-DRB1*09:01 and HLA-DRB1*14:03 alleles [47-49]. It was also interesting to find that DRB1*08:03, HLA-DRB1*09:01 and HLA-DRB1*14:03 were associated with GD in Asian populations $[14,20,22,50]$. Future studies should focus on which loci are attributable to GD maintenance and development.
In the present meta-analysis, heterogeneity was a potential problem when interpreting the results. Statistically significant heterogeneity was observed between studies for HLA-B*46 $\left(\mathrm{P}_{\text {heterogeneity }}<0.01, \mathrm{I}^{2}=\right.$ $68.0 \%)$. Between-study heterogeneity may be due to the different geographical regions of subjects. All subjects in current meta-analysis study were Asians, but from different regions and not a genetically homogenous group. Thus, this might explain the significant between-study heterogeneity. The differences in age and gender distributions among the included studies, and differences in the sample content and the methods of HLA typing HLA might also have contributed to the heterogeneity. In addition, inconsistency in inclusion criteria to be case population among studies might be another reason of heterogeneity, for five of fourteen studies just mentioned the cases were Graves' disease cases, not included the diagnostic criteria [12, 14, 18, 22, 27].

There were some limitations to the meta-analysis in the current study. First, it is possible that a few studies, showing no association between HLA-B ${ }^{*} 46$ and GD, were not included in current meta-analysis, because some published studies did not report their HLA-B*46 allele data [51, 52]. Secondly, three studies used genotyping methods for HLA typing, while 11 studies used serological methods due to the limitations of immunological detection technology at the time of the studies. The different typing methods (serological and genotyping methods) might lead to a heterogeneity in current meta-analysis. 
In summary, in the present study we performed a meta-analysis on the association of GD with the HLA-B*46 in Asian populations. Our results indicated a significant association between GD and HLA-B*46 $(\mathrm{OR}=2.48)$. Confirmation of the HLA-B ${ }^{*} 46$ association with GD in Asians is fundamental for studies on GD complications, such as periodic paralysis, ophthalmopathy, or GD recurrence. A previous study reported that HLA-B ${ }^{*} 46$ was associated with GD complications in Asians, such as periodic paralysis [12]. In addition, identification of population-specific HLA alleles of GD is a critical first step for individualized medicine in the future.

\section{Acknowledgments}

This work was supported by grants from the Foundation of Yunnan Provincial Education Department (No. 09C0296), the Specific Association Foundation Program of Yunnan Provincial Science and Technology Department and Kunming Medical University (No. 2011FB226), the Yunnan Provincial Science and Technology Department (2008CC021), and National Natural Science Foundation of China (No. 30900798).

\section{Competing Interests}

The authors have declared that no competing interest exists.

\section{References}

1. Weetman AP. Graves' disease. N Engl J Med. 2000;343:1236-48.

2. Jacobson DL, Gange SJ, Rose NR, Graham NM. Epidemiology and estimated population burden of selected autoimmune diseases in the United States. Clin Immunol Immunopathol. 1997;84:223-43.

3. Tunbridge WM, Evered DC, Hall R, et al. The spectrum of thyroid disease in a community: the Whickham survey. Clin Endocrinol (Oxf). 1977;7:481-93.

4. Shapira Y, Agmon-Levin N, Shoenfeld Y. Defining and analyzing geoepidemiology and human autoimmunity. J Autoimmun. 2010;34:J168-77.

5. Stenszky V, Kozma L, Balazs C, Rochlitz S, Bear JC, Farid NR. The genetics of Graves' disease: HLA and disease susceptibility. J Clin Endocrinol Metab. 1985;61:735-40.

6. Prabhakar BS, Bahn RS, Smith TJ. Current perspective on the pathogenesis of Graves' disease and ophthalmopathy. Endocr Rev. 2003;24:802-35.

7. Yanagawa $\mathrm{T}$, Mangklabruks A, Chang $\mathrm{YB}$, et al Human histocompatibility leukocyte antigen-DQA1*0501 allele associated with genetic susceptibility to Graves' disease in a Caucasian population. J Clin Endocrinol Metab. 1993;76:1569-74.

8. Yanagawa T, Mangklabruks A, DeGroot LJ. Strong association between HLA-DQA1*0501 and Graves' disease in a male Caucasian population. J Clin Endocrinol Metab. 1994;79:227-9.

9. Uno H, Sasazuki T, Tamai H, Matsumoto H. Two major genes, linked to HLA and Gm, control susceptibility to Graves' disease. Nature. 1981;292:768-70.

10. Barlow AB, Wheatcroft N, Watson P, Weetman AP. Association of HLA-DQA1*0501 with Graves' disease in English Caucasian men and women. Clin Endocrinol (Oxf). 1996;44:73-7.

11. Zamani M, Spaepen M, Bex M, Bouillon R, Cassiman JJ. Primary role of the HLA class II DRB1*0301 allele in Graves disease. Am J Med Genet. 2000;95:432-7.
12. Hawkins BR, Ma JT, Lam KS, Wang CC, Yeung RT. Association of HLA antigens with thyrotoxic Graves' disease and periodic paralysis in Hong Kong Chinese. Clin Endocrinol (Oxf). 1985;23:245-52.

13. Naito S, Sasaki H, Arakawa K. Japanese Graves' disease: association with HLA-Bw46. Endocrinol Jpn. 1987;34:685-8.

14. Yeo PP, Chan SH, Thai AC, et al. HLA Bw46 and DR9 associations in Graves' disease of Chinese patients are age- and sex-related. Tissue Antigens. 1989;34:179-84.

15. Inoue D, Sato K, Enomoto T, et al. Correlation of HLA types and clinical findings in Japanese patients with hyperthyroid Graves' disease: evidence indicating the existence of four subpopulations. Clin Endocrinol (Oxf). 1992;36:75-82.

16. Inoue $\mathrm{D}$, Sato $\mathrm{K}$, Maeda $\mathrm{M}$, et al. Genetic differences shown by HLA typing among Japanese patients with euthyroid Graves' ophthalmopathy, Graves' disease and Hashimoto's thyroiditis: genetic characteristics of euthyroid Graves' ophthalmopathy. Clin Endocrinol (Oxf). 1991;34:57-62.

17. Dong RP, Kimura A, Okubo R, et al. HLA-A and DPB1 loci confer susceptibility to Graves' disease. Hum Immunol. 1992;35:165-72.

18. Chan SH, Lin YN, Wee GB, Ren EC, Lui KF, Cheah JS. Human leucocyte antigen DNA typing in Singaporean Chinese patients with Graves' disease. Ann Acad Med Singapore. 1993;22:576-9.

19. Onuma H, Ota M, Sugenoya A, Inoko H. Association of HLA-DPB1*0501 with early-onset Graves' disease in Japanese. Hum Immunol. 1994;39:195-201.

20. Cavan DA, Penny MA, Jacobs $\mathrm{KH}$, et al. The HLA association with Graves' disease is sex-specific in Hong Kong Chinese subjects. Clin Endocrinol (Oxf). 1994;40:63-6.

21. Ohtsuka K, Nakamura Y. Human leukocyte antigens associated with hyperthyroid Graves ophthalmology in Japanese patients. Am J Ophthalmol. 1998;126:805-10.

22. Huang SM, Wu TJ, Lee TD, Yang EK, Shaw CK, Yeh CC. The association of HLA -A, -B, and -DRB1 genotypes with Graves' disease in Taiwanese people. Tissue Antigens. 2003;61:154-8.

23. Chen PL, Fann CS, Chu CC, et al. Comprehensive genotyping in two homogeneous Graves' disease samples reveals major and novel HLA association alleles. PLoS One. 2011;6:e16635.

24. Cho WK, Jung MH, Choi EJ, Choi HB, Kim TG, Suh BK. Association of HLA alleles with autoimmune thyroid disease in Korean children. Horm Res Paediatr. 2011;76:328-34.

25. Tan S-H, Chan S-H, Lee B-W, Wee G-B, Wong H-B. HLA association in Singapore children with Grave's disease. Metabolism. 1988;37:518-9.

26. Tamai H, Tanaka K, Komaki G, et al. HLA and thyrotoxic periodic paralysis in Japanese patients. J Clin Endocrinol Metab. 1987;64:1075-8.

27. Chan SH, Yeo PP, Lui KF, et al. HLA and thyrotoxicosis (Graves' disease) in Chinese. Tissue Antigens. 1978;12:109-14.

28. Lee YJ, Huang CY, Ting WH, et al. Association of an IL-4 gene haplotype with Graves disease in children: experimental study and meta-analysis. Hum Immunol. 2011;72:256-61.

29. Greenacre MJ, Degos L. Correspondence analysis of HLA gene frequency data from 124 population samples. Am J Hum Genet. 1977;29:60-75.

30. Lau J, Ioannidis JP, Schmid CH. Quantitative synthesis in systematic reviews. Ann Intern Med. 1997;127:820-6.

31. Higgins JP, Thompson SG, Deeks JJ, Altman DG. Measuring inconsistency in meta-analyses. BMJ. 2003;327:557-60.

32. Peters JL, Sutton AJ, Jones DR, Abrams KR, Rushton L. Comparison of two methods to detect publication bias in meta-analysis. JAMA. 2006;295:676-80.

33. Begg CB, Mazumdar M. Operating characteristics of a rank correlation test for publication bias. Biometrics. 1994;50:1088-101.

34. Yeo PP, Chan SH, Lui KF, Wee GB, Lim P, Cheah JS. HLA and thyrotoxic periodic paralysis. Br Med J. 1978;2:930.

35. Singer DS, Mozes E, Kirshner S, Kohn LD. Role of MHC class I molecules in autoimmune disease. Crit Rev Immunol. 1997;17:463-8.

36. Farid NR, Stone E, Johnson G. Graves' disease and HLA: clinical and epidemiologic associations. Clin Endocrinol (Oxf). 1980;13:535-44.

37. Abecasis GR, Noguchi E, Heinzmann A, et al. Extent and distribution of linkage disequilibrium in three genomic regions. Am J Hum Genet. 2001;68:191-7.

38. Reich DE, Cargill M, Bolk $\mathrm{S}$, et al. Linkage disequilibrium in the human genome. Nature. 2001;411:199-204.

39. Stephens JC, Schneider JA, Tanguay DA, et al. Haplotype variation and linkage disequilibrium in 313 human genes. Science. 2001;293:489-93.

40. Bodmer WF. Evolutionary significance of the HL-A system. Nature. 1972;237:139-45. 
41. Carrington M, Stephens JC, Klitz W, Begovich AB, Erlich HA, Mann D. Major histocompatibility complex class II haplotypes and linkage disequilibrium values observed in the CEPH families. Hum Immunol. 1994;41:234-40.

42. Diilali-Saiah I, Benini V, Daniel S, Assan R, Bach JF, Caillat-Zucman S. Linkage disequilibrium between HLA class II (DR, DQ, DP) and antigen processing (LMP, TAP, DM) genes of the major histocompatibility complex. Tissue Antigens. 1996;48:87-92.

43. Sanchez-Mazas A, Djoulah S, Busson M, et al. A linkage disequilibrium map of the MHC region based on the analysis of 14 loci haplotypes in 50 French families. Eur J Hum Genet. 2000;8:33-41.

44. Dawkins R, Leelayuwat C, Gaudieri S, et al. Genomics of the major histocompatibility complex: haplotypes, duplication, retroviruses and disease. Immunol Rev. 1999;167:275-304.

45. Ahmad T, Neville M, Marshall SE, et al. Haplotype-specific linkage disequilibrium patterns define the genetic topography of the human MHC. Hum Mol Genet. 2003;12:647-56.

46. Ban Y, Davies TF, Greenberg DA, et al. Arginine at position 74 of the HLA-DR beta1 chain is associated with Graves' disease. Genes Immun. 2004;5:203-8.

47. Kupatawintu P, Pheancharoen S, Srisuddee A, Tanaka H, Tadokoro K, Nathalang O. HLA-A, -B, -DR haplotype frequencies in the Thai Stem Cell Donor Registry. Tissue Antigens. 2010;75:730-6.

48. Saito S, Ota S, Yamada E, Inoko H, Ota M. Allele frequencies and haplotypic associations defined by allelic DNA typing at HLA class I and class II loci in the Japanese population. Tissue Antigens. 2000;56:522-9.

49. Tokunaga K, Ishikawa Y, Ogawa A, et al. Sequence-based association analysis of HLA class I and II alleles in Japanese supports conservation of common haplotypes. Immunogenetics. 1997;46:199-205.

50. Katsuren E, Awata T, Matsumoto C, Yamamoto K. HLA class II alleles in Japanese patients with Graves' disease: weak associations of HLA-DR and -DQ. Endocr J. 1994;41:599-603.

51. Tandon N, Mehra NK, Taneja V, Vaidya MC, Kochupillai N. HLA antigens in Asian Indian patients with Graves' disease. Clin Endocrinol (Oxf). 1990;33:21-6.

52. Tong ZH, Zhu G, Yan LX, Zhu J, Feng YG, Xu G. Association of HLA with Graves' disease in Chinese. Chin Med J (Engl). 1987;100:296-8. 